\title{
Subfractions of high-density lipoprotein (HDL) and dysfunctional HDL in chronic kidney disease patients
}

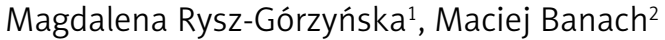

\begin{abstract}
${ }^{1}$ Department of Nephrology, Hypertension and Family Medicine, Healthy Aging Research Center, Medical University of Lodz, Lodz, Poland

${ }^{2}$ Department of Hypertension, Medical University of Lodz, Lodz, Poland
\end{abstract}

Submitted: 14 April 2016

Accepted: 28 April 2016

Arch Med Sci 2016; 12, 4: 844-849

DOI: $10.5114 /$ aoms.2016.60971

Copyright @ 2016 Termedia \& Banach

\section{Abstract}

A number of studies have shown that chronic kidney disease (CKD) is associated with increased risk for cardiovascular disease (CVD). Chronic kidney disease is characterized by significant disturbances in lipoprotein metabolism, including differences in quantitative and qualitative content of high-density lipoprotein (HDL) particles. Recent studies have revealed that serum HDL cholesterol levels do not predict CVD in CKD patients; thus CKD-induced modifications in high-density lipoprotein (HDL) may be responsible for the increase in CV risk in CKD patients. Various methods are available to separate several subclasses of HDL and confirm their atheroprotective properties. However, under pathological conditions associated with inflammation and oxidation, HDL can progressively lose normal biological activities and be converted into dysfunctional $\mathrm{HDL}$. In this review, we highlight the current state of knowledge on subfractions of HDL and HDL dysfunction in CKD.

Key words: high-density lipoprotein (HDL), subfractions, dysfunctional HDL, chronic kidney disease.

\section{Introduction}

Chronic kidney disease (CKD) affects approximately $16 \%$ of the general population, and this figure is projected to rise [1]. Chronic kidney disease is accompanied by very high cardiovascular mortality due to accelerated atherosclerosis [2]. In addition, declining kidney function has been identified as a strong cardiovascular (CV) risk factor [3, 4]. Cardiovascular damage starts early in the development of renal disease, and even mild kidney dysfunction may be an independent predictor for cardiovascular disease (CVD) or stroke [5, 6]. Moreover, some studies have demonstrated that in CKD patients left ventricular diastolic dysfunction occurs frequently and is associated with coronary artery disease and higher mortality [7]. Chronic kidney disease is an inflammatory state characterized by quantitative and qualitative alterations of the plasma lipids. It involves all lipoprotein classes and shows considerable variations depending on the stage of CKD. The causes of disturbances in lipoprotein metabolism are complex and depend on the rate of decline of the glomerular filtration rate (GFR) [8]. The lipid profile of CKD patients is typified by lower serum concentration of high-density lipoproteins $(\mathrm{HDL})$, higher serum concentrations of triglycerides (TG), apolipoprotein B

\author{
Corresponding author: \\ Magdalena Rysz-Górzyńska \\ Department of Nephrology, \\ Hypertension and \\ Family Medicine \\ Medical University of Lodz \\ 113 Zeromskiego St \\ 90-549 Lodz, Poland \\ Phone: +48 426393750 \\ Fax: +48426393782 \\ E-mail: mrs-89@o2.pl
}


(apoB), lipoprotein(a) [Lp(a)], remnant intermediate density lipoproteins (IDL) and very-low-density lipoproteins (VLDL), and an elevated proportion of oxidized low-density lipoproteins (oxLDL) [9, 10]. The low-density lipoprotein (LDL) cholesterol level is not usually raised, and it may even be decreased. This heterogeneity in patients with CKD results from differences in quantitative and qualitative content of lipids, apolipoproteins (apos), lipid transfer proteins and enzymes, which directly affect their biological activity and metabolism [11]. High serum LDL cholesterol levels are associated with CV risk in the general population as well as in the CKD population. It is well known that statins exert a beneficial effect on the kidney. Lipid-lowering agents are associated with cardiovascular and anti-proteinuric benefits in CKD patients [12-15]. Recent studies have shown that serum HDL cholesterol levels do not predict CVD in CKD patients; thus CKD-induced modifications in HDL may support the increase in CV risk in CKD patients.

\section{High-density lipoprotein subfractions}

High-density lipoproteins (HDL) are spherical micelles, with a density of $1.063-1.210 \mathrm{~g} / \mathrm{ml}$ and a small diameter $(7.5-10 \mathrm{~nm})$. All the components of $\mathrm{HDL}$ are convertible; thus traditional methods such as X-ray crystallography or nuclear magnetic resonance imaging are useless in identification [16]. The functional heterogeneity of HDL makes their comprehensive characterization quite challenging for investigators, who are searching for more practical and helpful laboratory methods [17]. High-density lipoproteins particles are composed of cholesteryl esters and triglycerides, which fill the hydrophobic core, and apolipoproteins, phospholipids and unesterified cholesterol forming the outer layer [18]. The main apolipoproteins associated with $\mathrm{HDL}$ are apolipoprotein A-I (apoA-I) and apolipoprotein A-II (apoA-II), supported by minor apolipoproteins (apoE, apoC-I, apoC-II, apoC-III, apoC-IV, apoC-V). High-density lipoproteins particles are heterogeneous lipoproteins carrying a large variety of enzymes, globulins, microRNAs, complement components and acute phase reactants [19]. The enzymes responsible for an antioxidant effect are paraoxonase-1 (PON1), platelet-activating factor acetyl hydrolase (PAF-AH), glutathione selenoperoxidase (GSPx), phospholipid transfer protein (PLTP) and lecithin-cholesterol acyltransferase (LCAT) [20, 21].

High-density lipoproteins are extremely complex and have been classified according to size, density, electrophoretic mobility and apo composition. Different subfractions of HDL particles can be distinguished using various techniques. By density gradient ultracentrifugation, the earliest laboratory method, two main subfractions,
HDL2 (larger and less dense) and HDL3 (smaller and more dense), can be separated [22]. It is still controversial which of the two has greater anti-atherogenic potency. Maeda et al. [23] found that subjects with a higher concentration of HDL2 were less likely to develop atherosclerosis. Also Kasko et al. [24] observed an increase of the small HDL3 subclass in individuals with newly diagnosed lower extremity artery disease (LEAD) without diabetes mellitus and without hypolipidemic therapy, which suggests that HDL3 is a potentially proatherogenic subclass. Conversely, the analysis of the Framingham Offspring Study (FOS) revealed that HDL-3 was protective and associated with decreased CV risk, while there was no significant association between HDL2 and CV risk. Using gradient gel electrophoresis, HDL particles can be further divided into HDL2b, HDL2a, HDL3a, HDL3b and HDL3c subclasses [25] (HDL2b has the largest diameter and HDL3c the smallest). In addition, HDLs have been classified according to both electrophoretic mobility and size using two-dimensional gel electrophoresis, which allows more than 10 subspecies to be distinguished [26]. $2 \mathrm{D}$ electrophoresis, the most informative method, separates lipid-poor pre- $\beta-\mathrm{HDL}$ and cholesterol ester-containing $\alpha$-HDL. Moreover, pre- $\beta 1-\mathrm{HDL}$ (very small, rich in apo- 1 ), pre- $\beta 2-\mathrm{HDL}$, pre- $\beta 3-\mathrm{HDL}$ (large), $\alpha 4-\mathrm{HDL}$ (very small, discoid, containing apoA-I, phospholipids and free cholesterol), small spherical $\alpha 3-H D L$ (containing apoA-I, apoA-II, phospholipids, free cholesterol, cholesteryl ester and triglyceride, medium size), spherical $\alpha 2-H D L$, which contains the same constituents as $\alpha 3-\mathrm{HDL}$, and large, spherical $\alpha 1-H D L$, with the same components as $\alpha 3$ - and $\alpha 2-H D L$ except the absence of apoA-II and pre- $\alpha$ (pre- $\alpha 1$, pre- $\alpha 2$ and pre- $\alpha 3$, with similar size as $\alpha$-particles, but without apoA-II) can be identified [27]. Asztalos et al. [28] reported a significant association between lower serum concentrations of $\alpha 1$ and $\alpha 2$ and higher $\alpha 3-\mathrm{HDL}$ level and elevated risk for new CVD events.

The division of HDL into separate lipoprotein particles can be achieved using anti-apolipoprotein immunoaffinity chromatography. Immunoseparation divides HDLs into a fraction containing only apoA-I (LPAI) and a fraction containing both apolipoproteins (LpAl:All), which are the two most abundant HDL particles. High density lipoproteins containing only apoE (LpE) and apoA-II (LpE:AII) are minor lipoprotein particles but also important [29, 30].

Another electrophoretic approach to study HDL heterogeneity is the LipoPrint system (Quantimetrix), which uses a non-denaturing polyacrylamide gel to separate subfractions from pre-stained serum or plasma lipids. The method is approved by the Food and Drug Administration (FDA) as a diagnostic technique for lipoprotein subfraction inves- 
tigation in the USA [31]. High density lipoproteins subclasses are divided into ten bands (HDL1-10), which form three groups: large (HDL1-3), intermediate (HDL4-7) and small (HDL8-10) particles. Oravec et al. [32] proposed that small HDL subfractions (HDL8-10) might have impaired functionality and that there is a correlation between higher concentrations of the small HDL subpopulation and atherogenic lipid profile.

In addition, a novel, gas-phase differential electrophoretic macromolecular mobility based method - ion mobility - has been designed to separate HDL2b from smaller HDL. This method revealed that large HDL2b subspecies inversely correlate with coronary artery disease (CAD) in the prospective Malmo Diet and Cancer Study [33]. The classification and quantitation of $\mathrm{HDL}$ particles may also be performed using nuclear magnetic resonance (NMR) spectroscopy [34]. Due to the specific physical structure and natural magnetic distinctness of HDLs, NMR spectroscopy does not require physical fractionation. Although the NMR method currently accounts for 26 different-size $\mathrm{HDL}$ subfractions, the measurement precision is limited, and only three HDLs are reported (large, medium and small) [35].

Because of various physical methods used in measurement of lipoproteins, an integrated nomenclature has recently been proposed: very large, large, medium, small and very small HDL particles, including discoid pre- $\beta$-HDL [35].

\section{Dysfunctional high-density lipoprotein}

Clinical and experimental evidence suggests that the association between individual HDL subclasses and coronary heart disease risk is complex. For years, many authors have suggested that larger HDL particles are more atheroprotective, but recent studies have demonstrated conflicting data [27]. The situation is also complicated in patients with CKD or end-stage renal disease (ESRD). In the study of Alabakovska et al. [36], in patients with ESRD, HDL2b (very large) particles were reduced and HDL3c (very small) particles were much more prevalent. On the other hand, Soto-Miranda et al. [37] observed a shift in HDL size distribution towards large particles in patients with proteinuria. Recent studies have shown that levels of the HDL3 subfraction decreased along with increasing CKD severity [38]. Diverse laboratory methods confirmed different HDL subfractions as being anti-atherogenic or associated with decreased CV risk. Nevertheless, HDL particles are not always vasoprotective, and pathological conditions such as inflammation, oxidative stress or diabetes can impair their functionality [39-41]. The vasoprotective properties of HDL have been exerted by reverse cholesterol transport (RCT). High density lipoproteins shuttles excess cholesterol and phospholipids from peripheral cells and macrophages to the liver, where they are excreted by the biliary route [42]. Chronic kidney disease reduces expression of ABCA1 (ATP-binding cassette transporter), which promotes the efflux of cholesterol from macrophages to apoA-1. The major protein in $H D L$, apoA-1, is decreased in patients with kidney disease because of diminished gene expression in the liver and deficiency of one of the constituent enzymes of HDL - lecithin cholesterol acetyltransferase (LCAT) [43]. The LCAT plays a major role in the formation of HDL by esterification of free cholesterol on the surface of HDL and its sequestration in the core of the molecule. Thus, LCAT deficiency, due to CKD-mediated down-regulation in the liver, hinders the uptake and maturation of HDL [44]. The study by Yamamoto et al. indicated that patients with CKD demonstrate abnormal HDL capacity to mediate cholesterol efflux, and it was significantly decreased compared with HDL from healthy subjects [45]. Moreover, Holzer et al. [46] observed that the efflux capacity of HDL in patients with ESRD was reduced compared with HDL from controls without renal disease. Patients with stages 3-4 CKD showed a progressive reduction in cholesterol efflux capacity of HDL [47]. There are numerous observations demonstrating anti-inflammatory, antithrombotic and antioxidant activities of HDL particles [48]. High-density lipoproteins from healthy subjects stops LDL oxidation and inhibits the expression of vascular cell adhesion protein 1 (VCAM-1), intercellular adhesion molecule (ICAM-1) and E-selectin in endothelial cells, which are responsible for infiltration of vascular walls by monocytes and macrophages, leading to atherosclerosis $[49,50]$. However, antiinflammatory activity may also be affected by systemic conditions, and under pathological conditions, such as inflammation or oxidative stress, $\mathrm{HDL}$ is referred to as dysfunctional HDL [51]. After post-transitional changes, which impair the anti-atherogenic function, dysfunctional HDL can even promote the production of inflammatory cytokines. Weichhart et al. [52] reported that HDL from healthy subjects inhibited the output of in flammatory cytokines (IL-10, IL-12, TNF- $\alpha$ ), while $\mathrm{HDL}$ from ESRD patients promoted the production of inflammatory cytokines.

Due to the activities of HDL-associated enzymes (lipoprotein-associated phospholipase A2 (Lp-PLA2), LCAT, paraoxonase (PON), platelet-activating factor acetylhydrolase (PAF-AH), glutathione peroxidase (GPX) and apoprotein components (apoprotein A-I and A-II), HDL plays an antioxidant role, including prevention of $L D L$ oxidation [53-55]. Many studies have indicated that apoA-I (most among all apos) mediates antioxidant activ- 
ities by removing oxidized phospholipids from LDL and from endothelium [56]. Systemic oxidative stress, which is very common in CKD patients, has been shown to reduce antioxidant and anti-inflammatory effects of HDL and even to convert it into a pro-oxidant and pro-inflammatory agent $[57,58]$. During chronic inflammation, the concentration of leukocyte myeloperoxidase (MPO) is increased. Zheng et al. [59] reported that MPO altered the function of HDL, especially apoA-I. Moradi et al. [60], who analyzed diminished antioxidant activity of HDL, noted reductions of apo-Al (41\%), LCAT (60\%), GPX (50\%) and PON (30\%) levels in ESRD patients compared to healthy subjects. These results were accompanied by a $127 \%$ reduction in $\mathrm{HDL}$ antioxidant activity. Even in moderate CKD, PON activity is diminished and correlates with non-fatal myocardial infarction, stroke and death [61]. Moreover, apoA-I oxidation decreased its interaction with ABCA1 and inhibited cholesterol efflux from macrophages [62]. Apparently, HDL exerts an antithrombotic effect through reducing platelet aggregation and thrombus formation. HDL was found to inhibit secretion of thromboxane A, which is mediated via scavenger receptor type B1 and/or the apolipoprotein E receptor apoER2/LRP8 [63]. In addition, HDL has a different mechanism contributing to antithrombotic effects. Due to the delivery of arachidonic acid to artery wall cells, which plays a pivotal role in prostacyclin (PGI2) synthesis, HDL improves its antithrombotic value. Prostacyclin has been shown to regulate the release of growth factor and to inhibit platelet activation [64]. High-density lipoproteins modified by an inflammatory response is unable to reduce thrombus formation. High-density lipoproteins is also known to stimulate fibrinolysis by reducing the production of plasminogen activator inhibitor-1 (PAI-1), which then increases the synthesis of tissue plasminogen activator (tPA) and the output of plasmin. High-density lipoproteins of CKD patients has shown impairment in profibrinolytic function [65]. Several studies have reported that $\mathrm{HDL}$ mediates endothelial cell proliferation, migration and adhesion molecule formation [66, 67]. Interestingly, these functions of HDL in CKD patients become profoundly depressed. Speer et al. [68] observed significantly decreased stimulation of endothelial cell proliferation and impaired ability to promote endothelial cell survival and repair in children and adults with CKD.

To sum up, these studies clearly indicate that HDL particles can lose their normal biological activities and acquire impaired properties as a result of perturbations in metabolism and composition. These alterations in HDL structure are characteristic for CKD and other pathological conditions associated with inflammation, infection or oxida- tive stress [69]. Chronic kidney disease, especially in advanced stages, affects the ability of HDL to accept free cholesterol and phospholipids from peripheral tissues, to control inflammation and oxidation, and to support the endothelium.

In conclusion, available data obtained using various laboratory techniques employed to describe plasma HDL support the heterogeneity of HDL particles. Further analysis of HDL particles' composition may explain biological functions of HDL subclasses, including those with altered properties. Alterations in HDL subpopulations in CKD patients not only predict CV risk but also accelerate the development of atherosclerosis. Even though the findings of recent studies have widened our view of HDL subclasses, there is still a lack of research performed on patients with CKD.

Most clinical studies concentrate on the quantity of HDL, and not on the quality and functionality of its subpopulations. Available data confirm that HDLs of CKD patients become deprived of anti-inflammatory, antioxidant and vasoprotective properties. However, it is still unknown whether size, density or functional alteration causes their dysfunctionality. Further clinical investigations are required to evaluate which subclasses are atheroprotective and which tend to become dysfunctional in patients with CKD.

\section{Acknowledgments}

The study was financed by the Polish National Science Centre (OPUS Grant, contract No. DEC2013/09/B/NZ5/02746).

\section{Conflict of interest}

The authors declare no conflict of interest.

\section{References}

1. Levey AS, de Jong PE, Coresh J, et al. The definition, classification, and prognosis of chronic kidney disease: a KDIGO Controversies Conference report. Kidney Int 2011; 80: 17-28.

2. Gluba-Brzózka A, Michalska-Kasiczak M, Franczyk B, et al. Markers of increased atherosclerotic risk in patients with chronic kidney disease: a preliminary study. Lipids Health Dis 2016; 15: 22.

3. Mahmoodi BK, Matsushita K, Woodward M, et al. Chronic Kidney Disease Prognosis Consortium. Associations of kidney disease measures with mortality and end-stage renal disease in individuals with and without hypertension: a meta-analysis. Lancet 2012; 380: 1649-61.

4. Chronic Kidney Disease Prognosis Consortium Association of estimated glomerular filtration rate and albuminuria with all-cause and cardiovascular mortality in general population cohorts: a collaborative meta-analysis. Lancet 2010; 375: 2073-81.

5. Go AS, Chertow GM, Fan D, McCulloch CE, Hsu CY. Chronic kidney disease and the risks of death, cardio- 
vascular events, and hospitalization. N Engl J Med 2004; 351: 1296-305.

6. Van Biesen W, De Bacquer D, Verbeke F, Delanghe J, Lameire N, Vanholder R. The glomerular filtration rate in an apparently healthy population and its relation with cardiovascular mortality during 10 years. Eur Heart J 2007; 28: 478-83.

7. Franczyk-Skóra B, Gluba A, Olszewski R, Banach M, Rysz J. Clinical research: heart function disturbances in chronic kidney disease - echocardiographic indices. Arch Med Sci 2014; 10: 1109-6.

8. Thompson M, Ray U, Yu R, et al. Kidney function as a determinant of $\mathrm{HDL}$ and triglyceride concentrations in the Australian population. J Clin Med 2016; 5: pii: E35 doi: 10.3390/jcm5030035.

9. Chmielewski M, Carrero JJ, Nordfors L, Lindholm B, Stenvinkel P. Lipid disorders in chronic kidney disease: reverse epidemiology and therapeutic approach. J Nephrol 2008; 21: 635-44

10. Bowe B, Xie Y, Xian H, Balasubramanian S, Al-Aly Z. Low levels of high-density lipoprotein cholesterol increase the risk of incident kidney disease and its progression. Kidney Int 2016; 89: 886-96.

11. Otocka-Kmiecik A, Mikhailidis DP, Nicholls SJ, Davidson M, Rysz J, Banach M. Dysfunctional HDL: a novel important diagnostic and therapeutic target in cardiovascular disease? Prog Lipid Res 2012; 51: 314-24.

12. Rysz J, Gluba-Brzózka A, Banach M, Więcek A. Should we use statin in all patients with chronic kidney disease without dialysis therapy? The current state of knowledge. Int Urol Nephrol 2015; 47: 805-13.

13. Banach M, Aronow WS, Serban MC, Rysz J, Voroneanu L, Covic A. Lipids, blood pressure and kidney update 2015 Lipids Health Dis 2015; 14: 167.

14. Colantonio LD, Baber U, Banach M, et al. Contrasting cholesterol management guidelines for adults with CKD. J Am Soc Nephrol 2015; 26: 1173-80.

15. Toth PP, Barylski M, Nikolic D, Rizzo M, Montalto G, Banach $M$. Should low high-density lipoprotein cholesterol (HDL-C) be treated? Best Pract Res Clin Endocrino Metab 2014; 28: 353-68.

16. Pownall H, Rosales C, Gillard B, Moon J, Gotto A. Human plasma lipoprotein metabolism. In: Clinical Lipidology: A companion to Braunwald's heart disease. 2nd ed. Ballantyne C (ed.). Philadelphia 2015; 3.

17. Dragan S, Serban C, Banach M. Can we change the functionality of $\mathrm{HDL}$ cholesterol with non-pharmacological and pharmacological agents? Curr Med Chem 2014; 21 : 2927-46.

18. Asztalos, BF, Schaefer EJ. High-density lipoprotein subpopulations in pathologic conditions. Am J Cardiol 2003; 91: 12E-7E.

19. Toth PP, Barter PJ, Rosenson RS, et al. High-density lipoproteins: a consensus statement from the National Lipid Association. J Clin Lipidol 2013; 7: 484-525.

20. Weichhart T, Kopecky C, Kubicek M, et al. Serum amyloid $A$ in uremic HDL promotes inflammation. J Am Soc Nephrol 2012; 23: 934-47.

21. Skinner ER. High-density lipoprotein subclasses. Curr Opin Lipidol 1994; 5: 241-7.

22. Chapman MJ, Goldstein S, Lagrange D, Laplaud PM. A density gradient ultracentrifugal procedure for the isolation of the major lipoprotein classes from human serum. J Lipid Res 1981; 22: 339-58.

23. Maeda S, Nakanishi S, Yoneda M, et al. Associations between small dense LDL, HDL subfractions (HDL2, HDL3) and risk of atherosclerosis in Japanese-Americans. J Atheroscler Thromb 2012; 19: 444-52.
24. Kasko M, Gaspar L, Dukát A, Gavorník P, Oravec S. High-density lipoprotein profile in newly-diagnosed lower extremity artery disease in Slovak population without diabetes mellitus. Neuro Endocrinol Lett 2014 35: 531-5.

25. Farbstein D, Levy AP. HDL dysfunction in diabetes: causes and possible treatments. Expert Rev Cardiovasc Ther 2012; 10: 353-61.

26. Schaefer EJ, Santos RD, Asztalos BF. Marked HDL deficiency and premature coronary heart disease. Curr Opin Lipidol 2010; 21: 289-97.

27. Rizzo M, Otvos J, Nikolic D, Montalto G, Toth PP, Banach $M$. Subfractions and subpopulations of HDL: an update. Curr Med Chem 2014; 21: 2881-91.

28. Asztalos, BF, Collins D, Cupples LA, et al. Value of high-density lipoprotein (HDL) subpopulations in predicting recurrent cardiovascular events in the Veterans Affairs HDL Intervention Trial. Arterioscler Thromb Vasc Biol 2005; 25: 2185-91.

29. Alaupovic P. The concept of apolipoprotein-defined lipoprotein families and its clinical significance. Curr Atheroscler Rep 2003; 5: 459-67.

30. Duriez P, Fruchart JC. High-density lipoprotein subclass es and apolipoprotein A-I. Clin Chim Acta 1999; 286 97-114

31. Dukát A, Oravec S, Wawruch M, Gavorník P, Sabaka P. The incidence of dyslipidemia in a sample of asymptomatic probands established by the means of Lipoprint system. Vnitr Lek 2011; 57: 258-60.

32. Oravec S, Dukat A, Gavornik P, et al. Atherogenic versus non-atherogenic lipoprotein profiles in healthy individu als. Is there a need to change our approach to diagnosing dyslipidemia? Curr Med Chem 2014; 21: 2892-901.

33. Musunuru K, Orho-Melander M, Caulfield MP, et al. Ion mobility analysis of lipoprotein subfractions identifies three independent axes of cardiovascular risk. Arterioscler Thromb Vasc Biol 2009; 29: 1975-80.

34. Otvos JD, Collins D, Freedman DS, et al. Low-density lipoprotein and high-density lipoprotein particle subclasses predict coronary events and are favorably changed by gemfibrozil therapy in the Veterans Affairs High-Density Lipoprotein Intervention Trial. Circulation 2006; 113 1556-63.

35. Rosenson RS, Brewer HB Jr, Chapman MJ, et al. HDL measures, particle heterogeneity, proposed nomenclature, and relation to atherosclerotic cardiovascular events. Clin Chem 2011; 57: 392-410.

36. Alabakovska SB, Todorova BB, Labudovic DD, Toshes ka KN. LDL and HDL subclass distribution in patients with end-stage renal diseases. Clin Biochem 2002; 35: 211-6.

37. Soto-Miranda E, Carreón-Torres E, Lorenzo K, et al. Shift of high-density lipoprotein size distribution toward large particles in patients with proteinuria. Clin Chim Acta 2012; 414: 241-5.

38. Honda $\mathrm{H}$, Hirano T, Ueda M, et al. High-density lipoprotein subfractions and their oxidized subfraction particles in patients with chronic kidney disease. Atheroscler Thromb 2016; 23: 81-94.

39. Morgantini C, Natali A, Boldrini B, et al. Anti-inflammatory and antioxidant properties of HDLs are impaired in type 2diabetes. Diabetes 2011; 60: 2617-23.

40. Lagos KG, Filippatos TD, Tsimihodimos V, et al. Alterations in the high density lipoprotein phenotype and HDL-associated enzymes in subjects with metabolic syndrome. Lipids 2009; 44: 9-16. 
41. McGillicuddy FC, de la Llera MM, Hinkle C, et al. Inflammation impairs reverse cholesterol transport in vivo. Circulation 2009; 119: 1135-45.

42. Wang X, Rader DJ. Molecular regulation of macrophage reverse cholesterol transport Curr Opin Cardiol 2007; 22: 368-72.

43. Vaziri ND, Deng G, Liang K. Hepatic HDL receptor, SR-B1 and Apo A-I expression in chronic renal failure. Nephrol Dial Transplant 1999; 14: 1462-6.

44. Serban C, Muntean D, Mikhailids DP, Toth PP, Banach M. Dysfunctional HDL: the journey from savior to slayer. Clin Lipidol 2014; 9: 49-59.

45. Yamamoto S, Yancey PG, Ikizler TA, et al. Dysfunctiona high-density lipoprotein in patients on chronic hemodialysis. J Am Coll Cardiol 2012; 60: 2372-9.

46. Holzer M, Birner-Gruenberger R, Stojakovic T, et al. Uremia alters HDL composition and function. J Am SocNephrol 2011; 22: 1631-41.

47. Kaseda R, Fazio S, Kon V. Chronic kidney disease (CKD) disruption of macrophage lipid handling and inflammation and effect of liver $\mathrm{X}$ receptor (LXR) agonism. ASN Kidney Week 2014; 25: 869.

48. Nofer JR, Kehrel B, Fobker M, et al. HDL and arteriosclerosis: beyond reverse cholesterol transport. Atherosclerosis 2002; 161: 1-16.

49. Patel S, Di Bartolo BA, Nakhla S, et al. Anti-inflammatory effects of apolipoprotein A-I in the rabbit. Atherosclerosis 2010; 212: 392-7.

50. Barter PJ, Nicholls S, Rye KA, Anantharamaiah GM, Nav ab M, Fogelman AM. Antiinflammatory properties of HDL. Circ Res 2004; 95: 764-72.

51. Navab M, Hama SY, Hough GP, Subbanagounder G, Reddy ST, Fogelman AM. A cell-free assay for detecting HDL that is dysfunctional in preventing the formation of or inactivating oxidized phospholipids. J Lipid Res 2001; 42: 1308-17.

52. Weichhart T, Kopecky C, Kubicek M, et al. Serum amyloid $A$ in uremic HDL promotes inflammation. J Am Soc Nephrol 2012; 23: 934-47.

53. Toth PP. High-density lipoprotein: epidemiology, metabolism, and antiatherogenic effects. Dis Mon 2001; 47: 369-416.

54. Podrez EA. Anti-oxidant properties of high-density lipoprotein and atherosclerosis. Clin Exp Pharmacol Physio 2010; 37: 719-25.

55. Florentin M, Liberopoulos EN, Wierzbicki AS, Mikhailidis DP. Multiple actions of high-density lipoprotein. Curr Opin Cardiol 2008; 23: 370-8.

56. Navab M, Hama SY, Anantharamaiah GM, et al. Normal high density lipoprotein inhibits three steps in the formation of mildly oxidized low density lipoprotein: steps 2 and 3. J Lipid Res 2000; 41: 1495-508.

57. Van Lenten BJ, Reddy ST, Navab M, Fogelman AM. Understanding changes in high density lipoproteins during the acute phase response. Arterioscler Thromb Vasc Biol 2006; 26: 1687-8.

58. Kon V, Yang H, Fazio S. Residual cardiovascular risk in chronic kidney disease: role of high-density lipoprotein. Arch Med Res 2015; 46: 379-91.

59. Zheng L, Nukuna B, Brennan ML, et al. Apolipoprotein A-I is a selective target for myeloperoxidase catalyzed oxidation and functional impairment in subjects with cardiovascular disease. J Clin Invest 2004; 114: 529-41.

60. Moradi H, Pahl MV, Elahimehr R, Vaziri ND. Impaired an tioxidant activity of high-density lipoprotein in chronic kidney disease. Transl Res 2009; 153: 77-85.

61. Kennedy DJ, Tang WH, Fan Y, et al. Diminished antioxidant activity of high-density lipoprotein-associated pro- teins in chronic kidney disease. J Am Heart Assoc 2013; 2: e000104.

62. Shao B, Oda MN, Oram JF, Heinecke JW. Myeloperoxidase: an inflammatory enzyme for generating dysfunctional high density lipoprotein. Curr Opin Cardiol 2006; 21: 322-8.

63. Nofer JR, Brodde MF, Kehrel BE. High-density lipoproteins, platelets and the pathogenesis of atherosclerosis. Clin Exp Pharmacol Physiol 2010; 37: 726-35.

64. McGraw AP, Bagley J, Chen WS, et al. Aldosterone increases early atherosclerosis and promotes plaque inflammation through a placental growth factor-dependent mechanism. J Am Heart Assoc 2013; 2: e000018.

65. Mineo C, Deguchi H, Griffin JH, Shaul PW. Endothelial and antithrombotic actions of HDL. Circ Res 2006; 98 : 1352-64.

66. Riwanto M, Landmesser U. High density lipoproteins and endothelial functions: mechanistic insights and alterations in cardiovascular disease. J Lipid Res 2013; 54: 3227-43.

67. Moody WE, Edwards NC, Madhani M, et al. Endothelial dysfunction and cardiovascular disease in early-stage chronic kidney disease: cause or association? Atherosclerosis 2012; 223: 86-94.

68. Speer T, Rohrer L, Blyszczuk P, et al. Abnormal high-density lipoprotein induces endothelial dysfunction via activation of Toll-like receptor-2. Immunity 2013; 38: 754-68.

69. Kontush A, Chapman MJ. Functionally defective HDL: a new therapeutic agent at the cross roads of dyslipidemia, inflammation and atherosclerosis. Pharmacol Rev 2006; 3: 342-74. 